\title{
Design and experiments to investigate spray and impingement characteristics of a common rail type lubrication system
}

\author{
Matthias Stark*1, Arturo de Risi², Marcello Giangreco², Simon Diggelmann ${ }^{1}$ \\ ${ }^{1}$ WinGD, Winterthur Gas \& Diesel Ltd, Switzerland \\ ${ }^{2}$ University of Salento, Department of Engineering for innovation, Lecce, Italy \\ *Corresponding author: matthias.stark@wingd.com
}

\begin{abstract}
This paper reports on the establishment of a testing facility to investigate spray and impingement characteristics of a recently developed, fully adaptive lubrication system for large two stroke marine diesel engines. The reported part of this research relates to development steps towards the new lubrication system as well as the implementation of a dedicated testing facility and the establishment of validation approaches in order to corroborate technology developments in this context.
\end{abstract}

The major objective of this development yields an optimization of lubricant utilization and at the same time a significant reduction of lubricant fraction in the exhaust gas. A requisite to obtain the desired level of flexibility calls for a revised approach in injecting the lubricant. The desired flexibility of the new lubrication approach foresees a cycle based adjustment of the injected amount of lubricant as well as the injection pressure in order to provide the possibility to shape the injection spray pattern. Concept studies nominate the application of a common rail system with integrated needle lift type injectors and adjustable injection pressure as compliant with the requisite to inject the lubricant with a high level of flexibility.

This paper hence encompasses design and development aspects of the new lubrication system but most of all highlights steps in developing a validation concept in order to compare common lubrication systems with the new type of lubrication system.

Initial results of the test cell provide an insight regarding relevant information on the injection spray characteristics over the full engine load range as well as the possibility to compare common lubrication system performance with the new common rail lubrication system.

A comparison between measurement and simulation results provide the possibility to implement experimentally gained data in a neuronal network in order to enhance the predictive quality of the simulation tool.

\section{Keywords}

Injection system development and validation, computational injection spray simulation, experimental spray investigation

\section{Introduction}

Detailed investigations related to lubricant flow optimizations and transportation mechanisms clearly demonstrate the importance of an appropriate lubricant injection. [1-4] A characterization of relevant contributors to the total lubricant balance of a large two stroke marine diesel engine was performed to address the optimization potential of single components of the tribosystem. Optimizations of piston ring pack geometries showed superior functionality in terms of considerably reducing the amount of lubricant in the exhaust gas. Another, yet equal important aspect which must be taken into consideration when looking at further reducing lubricant consumption of such an engine type, relates to a detailed investigation regarding the application of the lubricant by means of a properly designed lubrication system. Design aspects of such a recent development focus on controlling lubricant spray characteristics over the complete engine load range and related variations of boundary conditions. Thus, investigations in the context of this paper, focus on developing an evaluation approach to support lubricant spray simulation tool developments as well as an experimental quantification of the lubrication system performance under close to real engine like boundary conditions.

One of the differences of a large two stroke diesel marine engine, compared to automotive engines, is found in the application of such a lubrication system. Electronically controlled lubrication systems are designed to meet 
appropriately defined lube oil feed rates in order to optimize cylinder lube oil consumption without compromising life time performance of a tribosystem. Mentioned lubrication systems deliver accurately metered, main engine load and fuel quality dependent quantities of lubricant to the cylinder liner with a very precise timing. This circumstance provides the possibility of adjusting lubrication system parameters on basis of operational requirements.

Fig. 1 shows a schematic of a "Pulse Jet" two stroke lubrication system, a typical setup of a lubrication system applied on WinGD's large two stroke marine diesel engines. It consists of a lubricant tank, a filter system, lube oil dosage pumps and non-return valve based lube oil injectors. The cylinder lube oil is applied onto the running surface of a cylinder liner by means of appropriately designed lube oil injector nozzle tips.

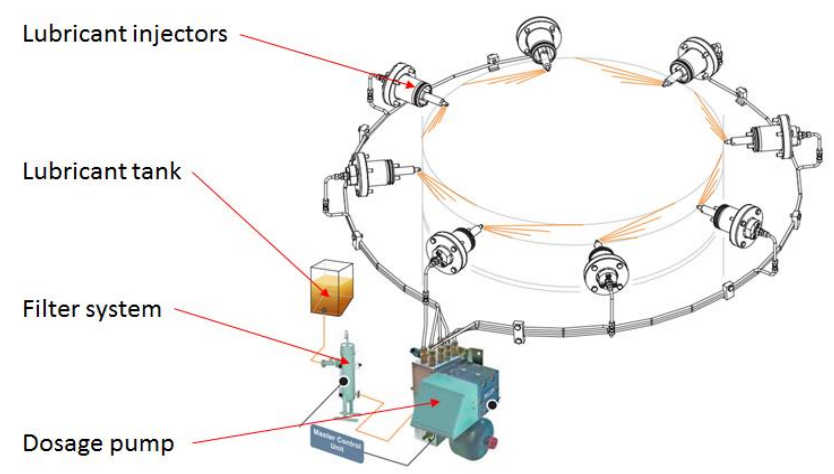

Fig.1: Typical arrangement of a large two stroke diesel engine lubrication system [2,3]

The lubricant is delivered to the injectors by a dosage pump which is powered by pressurized servo oil drawn from the engines system oil circuit. The lube oil feed rate and timing are electronically controlled by a solenoid valve of the dosage pump providing full flexibility in setting the injection timing over the full load range of the engine. A short coming of such a system is found in significant variations of the lubricant spray pattern following well investigated effects of a strong temperature dependency of the dynamic viscosity and related spray behaviour. A necessity to control the spray characteristics in order to optimize lubricant utilization thus leads to the development of a needle lift type in injection system in conjunction with a common rail approach.

\section{Lubrication system development}

Complying with the requirements of a fully adaptive lubrication system and the requisite to actively control lubricant spray characteristics over the entire engine load range, calls for a review of existing lubrication approaches. A significant part of this investigation hence focuses on the development of such a system to optimize lubricant utilization and to guarantee optimal life time performance. Fig.2 shows a schematic of the common rail type lubrication system incorporating needle lift type lubricant injectors.

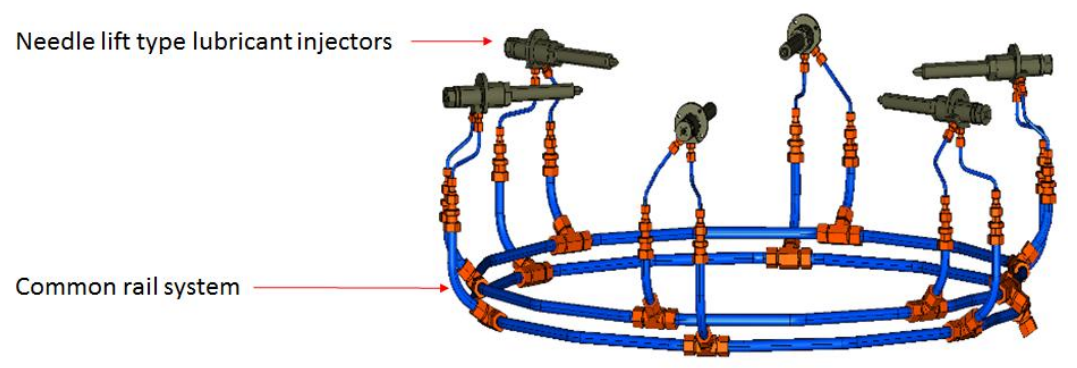

Fig.2: Schematic of a new common rail type lubrication system

Such an approach provides the possibility to adjust the injection pressure and timing over the complete engine load range and related temperature condition variations. Flexibility in shaping the lube oil spray pattern can thus achieved by applying main engine load dependent injection parameters. 
A typical development approach in this respect encompasses computational simulation support to optimize geometrical aspects and to predict lubrication system performance. A 1D fortran code based on concentrated volume method is applied to simulate the new lubricant injectors, taking friction effects on dynamics of moving components of the injector into consideration. Basic equations of the simulation tool are mass and momentum equations, whereof the integration of momentum equations on a solid body allows to model the moving parts, such as valves and needles. Forces due to the pressure on the surfaces, to the spring load and preload, to the friction forces were taken into account as well as the collision forces calculated by the elastic collision theory. An integral approach on a generic volume for the equation of mass is convenient when the main phenomenon is the pressure variation due to the flow rate and volume variation, as in the several chambers of the injection system. The discharge coefficient was evaluated according to Schmitt's formulation [14]; furthermore, the leakage between different chambers was evaluated considering laminar flow in the gap. Mass and momentum equations were employed to simulate the fluid dynamic phenomena inside the pipes and connections between different chambers of the system. Using a further relation between pressure and density, also provides the possibility to solve the equations in onedimensional form using the method of characteristics. $[9,11]$ This relation is based on the bulk modulus of the fluid, which is considered linear according to the pressure, interpolating the data obtained from measurements, the presence of air in the oil could also be considered. Nikuradse's theory was used to calculate the friction forces while the boundary conditions were calculated according to Matsuoka. All the equations were solved using an explicit first order Runge-Kutta scheme. A schematic of the simulated component is shown in Fig.3. a) and b) as explained by de Risi. [5]

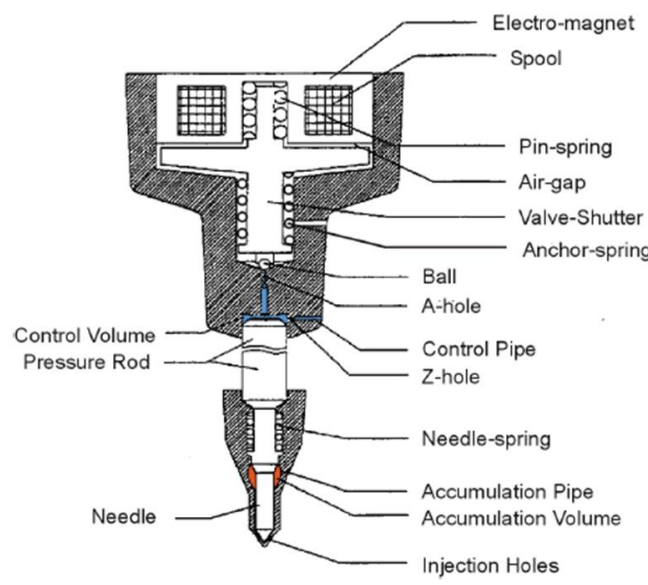

a)

Fig.3: a) Schematic of the simulated components in the LubeFluid 1D code; b) detailed view of the simulated scheme for the control zone, displaying the open position of a needle lift type injector. [5]

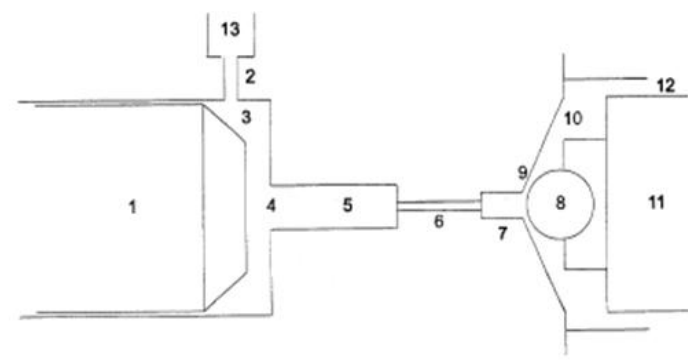

1) pressure rod; 2) Z-hole; 3) control volume; 4) A-hole entrance; 5) A-hole pre-chamber; 6) A-hole; 7) prehole; 8) ball; 9) valve seat; 10) ball-chamber; 11) valve actuator "pin"; 12) backflow pipes; 13) Zhole pre-chamber.

b)

A brief description of the new type of lubricant injector is described hereafter. In the upper part of the injector there is a magnetic circuit which is controlled by an Electronic Control Unit. When a voltage signal arrives from the ECU, the magnetic circuit attracts the valve-shutter and opens the valve. The control volume and the accumulation volume are high pressure chambers fed by the rail via adequate pipes. These chambers limit upwards and downwards the pressure rod. At rest (no voltage signal) both the control volume and the accumulation volume are at the same pressure and the pressure rod due to the different cross section at the bottom and at the top, keeps the needle in its seat. When the magnetic circuit open the valve, the pressure in the control volume gets lower since the fluid moves from this zone to the upper region through the open valve. As a consequence, the pressure rod is moved upwards by the pressure gradient, the needle is raised from its seat and the injection starts. When the electrical signal stops, the valve is closed and the injection ceases. The injection profile resulting from this process depends on the dynamic characteristic of the injector. In particular, the raising of the needle from its seat is governed by the fluid dynamics of both the control zone and the accumulation volume. The geometric features of these zones will be used as parameters in the optimization with genetic algorithms that will be carried out on basis of a first draft of the injector. [5]

Assuming a dependence of density on pressure only, it should be possible to introduce the fuel bulk modulus as defined by Parson [15]; moreover, the elasticity of the pipe walls has been calculated by reducing the bulk modulus of the fluid volume contained between them. The equations of flow inside a pipe were solved writing continuity and 
momentum equations considering one-dimensional isothermal flow. Taking into account those hypotheses, the equation system is:

$$
\begin{aligned}
& \left(\frac{\partial p}{\partial t}\right)+\left(\frac{u \partial p}{\partial x}\right)+\frac{\left(\rho c^{2} u \delta\right)}{A}+\rho c^{2}\left(\frac{\partial u}{\partial x}\right)=0 \\
& \left(\frac{\partial u}{\partial t}\right)+u\left(\frac{\partial u}{\partial x}\right)+\frac{\left(\frac{\partial p}{\partial x}\right)}{\rho}+f=0
\end{aligned}
$$

where $\delta=\left(\frac{d A}{d x}\right), u$ and $c$ are the oil and sound velocity, respectively, whereas $\rho$ is the fluid bulk density and $f$ is the friction factor.

Supposing that the density of both phases is only function of the pressure and does not vary, as the section pressure is constant and equal to the vapor pressure if there is the presence of a vapor phase, the continuity equation can be written as:

$$
\left(\rho_{v}-\rho_{l}\right) \frac{\partial \alpha}{\partial t}+u\left(\rho_{v}-\rho_{l}\right) \frac{\partial \alpha}{\partial x}+\rho \frac{\partial u}{\partial x}=0
$$

The pressure drop due to the friction in two-phase flow cannot be treated as mono-phase, since the vapor completely modifies the fluid dynamic conditions in the pipe; therefore, its evaluation was computed through the Lockhart's and Martinelli's empirical correlation. [13] A typical simulation result, obtained using the 1D LubeFluid code, is shown in Fig.4, which compares different injection timing effects on injection velocity profiles at 900 bar injection pressure.

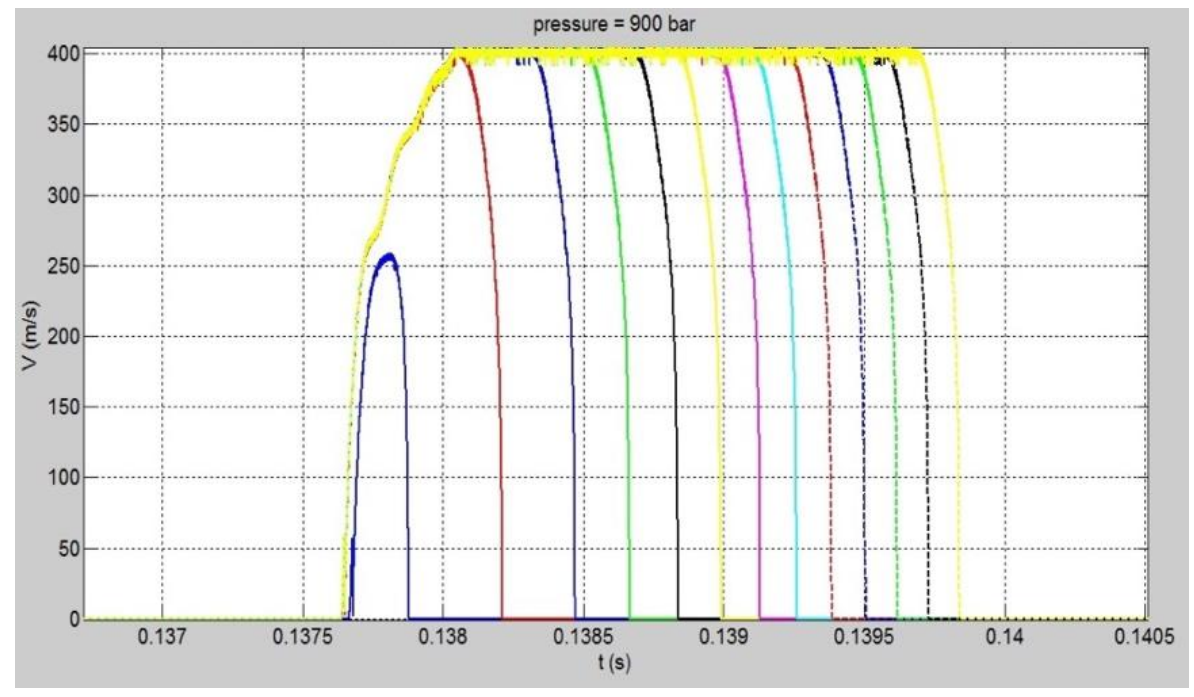

Fig.4: Injection velocity profile as function of excitation time. The first blue curve is representative of an injection in the ballistic regime. [5]

Initial simulation results highlight, that the time response of the injector significantly is affected by stiffness and preloading of its three springs: the pin spring, the anchor spring and the needle spring. These dynamic parameters hence are key parameters in the final optimization process to be performed in the next step of the ongoing project.

\section{Experimental setup}

Investigating mentioned injection characteristics calls for the establishment of a sound testing environment. Therefore a system was designed to provide testing conditions similar to real engine application boundary conditions in order to investigate thermo - physical properties and related effects on the lubricant spray. The test cell hence provides the possibility to address parameter variations such as pressure, temperature, injection pressure, nozzle geometry and lubricant property variations over the complete load range of a large two stroke marine diesel engine. 
The test cell design features a cylinder liner segment between two lubrication quill positions in order to simulate the lube oil spray under engine like conditions. Fig. 5 shows the segment which is covered and provided with an optical access in order to investigate on different effects on the lube oil spray pattern and the impingement characteristic on the cylinder liner wall.

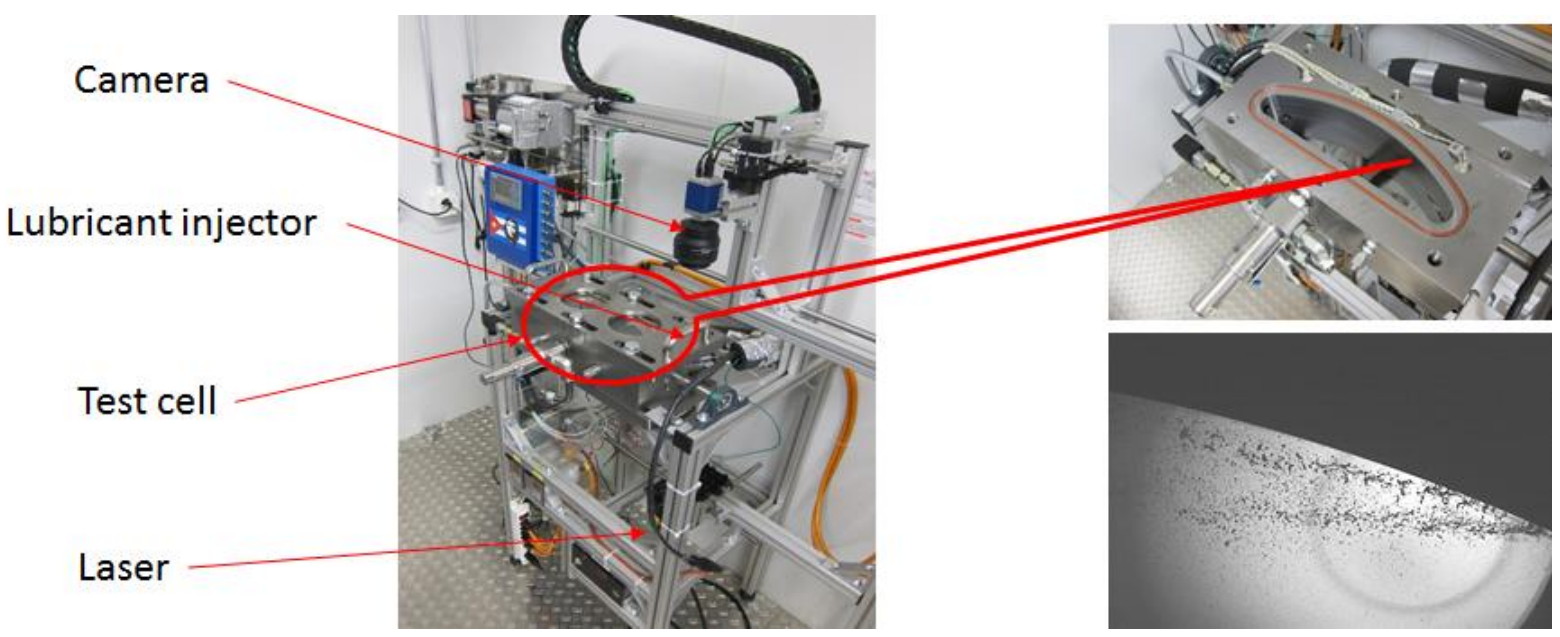

Fig.5: Schematic of the lube oil injection test cell and first impressions of the testing environment

In order to comply with real engine like conditions, a pre-study was performed to determine relevant system boundary parameters. The system is designed to provide pressure and temperature characteristics comparable to the full engine load range. Efforts in developing a fully operative test cell focus on the application of measurement technologies and instrumentation. A variety of Software and Hardware adaptions lead to the desired final test cell design. Adapting a standard Pulse Jet lubrication system and a high speed camera provides first impressions of a lube oil spray and gives a feedback on how the test cell performs.

Further adaptions of system components lead to preliminary information regarding the injection spray characteristics over the full engine load range. Fig. 6 shows a comparison between $25 \%$ and $100 \%$ engine load equivalent system boundary conditions and related lubricant spray patterns of a single-hole nozzle based on a typical diffused back-illumination measurement approach.
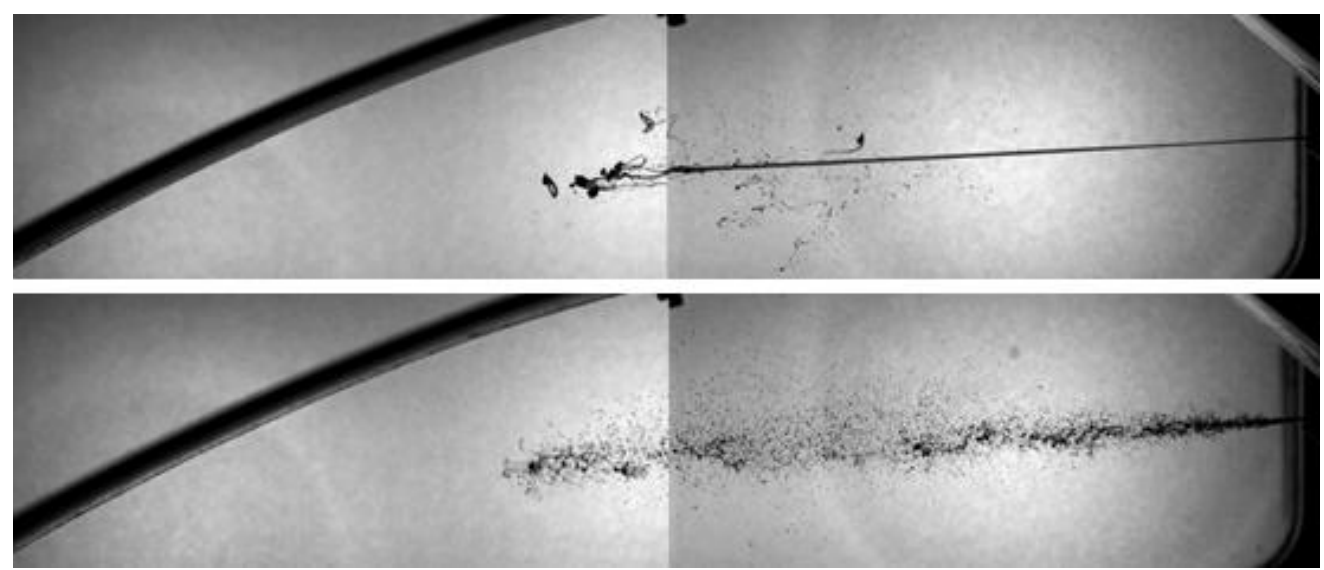

Fig.6: Diffused back-illumination approach measurement results of lube oil injection spray characteristics for 25\%engine load (1,6 bar of absolute cell pressure and $421 \mathrm{~K}$ lube oil temperature) and $100 \%$ engine load equivalent system boundary conditions (4.8 bar of absolute cell pressure and $476 \mathrm{~K}$ lube oil temperature)

Fig.6 also demonstrates the strong dependence between lube oil temperature and injection pressure. In fact, considering a lube oil temperature of $421 \mathrm{~K}$, an injection pressure of 1.6 bar absolute and a nozzle diameter of 0.75 $\mathrm{mm}$, the behaviour of the liquid phase is simply a liquid column. Instead, with an oil temperature of $476 \mathrm{~K}$ and an injection pressure of 4,8 bar absolute, a break-up in the liquid phase is visible, with the formation of a certain number of droplets. This break-up depends on the Ohnesorge number, which is the ratio between the Weber number square 
root and the Reynolds number. Thus, the decrease of the viscosity results in an increasing of the Reynolds number and a decay of the Ohnesorge number. The tested oil is characterized by an SAE 50 viscosity index with a kinematic viscosity of $19,2 \mathrm{~mm} / \mathrm{s}^{\wedge} 2 @ 100^{\circ} \mathrm{C}$ and for this reason the spray condition can shift from a no-break-up regime to a first wind induced break-up.

The acquired images are used to study spray characteristics. A Labview code is currently being developed to standardize measurement results for all testing conditions. Definitions of regions of interest (ROI) are utilized to characterize the development of the spray. Fig.7 shows a schematic of such an attempt in characterizing distinct injection characteristics. Two ROls are related to the upper and lower part of the spray (red and green rectangle) whereas a third one follows the spray tip along the distance from the nozzle towards the simulated cylinder wall. (yellow rectangle)

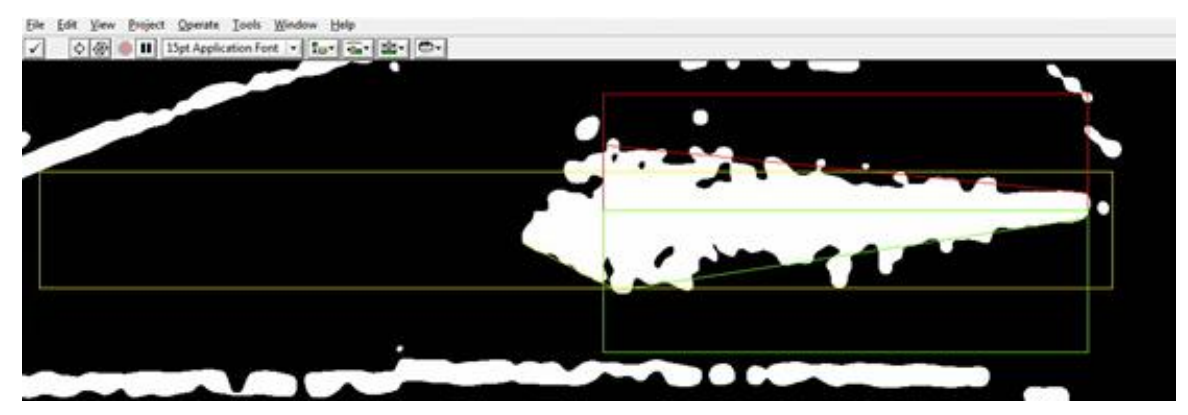

Fig.7: Schematic of a typical spray characterization approach

Next steps in this development relate to further optimizations of the system and the implementation of algorithms to quantify lubricant spray and impingement characteristics.

\section{Simulation tool development}

This section briefly discusses the numerical approach to develop a code which is capable of simulating lubricant sprays. A 1D CFD and a neural network approach is used with the objective to create a fast running application to compute spray characteristics variations and to quickly visualize the effect on the cylinder wall. A particular approach of predicting the lubricant spray characteristics has been applied by making use of a CFD numerical approach. Literature review and experimental data reveal the most suitable analytical spray models which best describe the phenomenology of lubricant sprays. [4]

The first step of the numerical code development relates to the computation of the jet velocity $v_{i}$ at the nozzle hole exit. This has been made using the relation derived from Bernoulli:

$$
v_{i}=k_{v} \sqrt{\frac{2 \Delta p}{\rho_{l}}}
$$

Where $k_{v}$ is the discharge coefficient from Hiroyasu et al, $\rho_{l}$ is the liquid phase density and $\Delta p$ is the pressure difference across the nozzle. Subsequently, the volume of the injected liquid for every time-step has been computed using a typical injection profile loaded from the acquired data, which is similar to the one in figure, to calculate the pressure-drop at every time-step (dt). Thus, the expression for the instant volume of oil is the following:

$$
V_{f}=A_{n z} v_{i} d t
$$

At this point it is necessary to compute the Sauter mean diameter (SMD) of the droplets so that it is possible to apply resulting drag forces. For this reason, a series of SMD prediction models has been implemented providing the possibility to change the model in the software graphic unit interface according to literature information related to the specific spray measurement results. The implemented models are derived from Hiroyasu-Kadota [6], Elkotb et al. [7], Hiroyasu-Arai [8], Hiroyasu-Arai-Tabata [9] respectively:

$$
S M D=23.9 \Delta p^{-0.135} \rho_{g}{ }^{0.121} V_{f}^{0.131}
$$




$$
\begin{gathered}
S M D=3.08 \Delta p^{-0.54} \rho_{g}{ }^{0.06}\left(\sigma \rho_{l}\right)^{0.737} v_{l}^{0.385} \\
S M D=0.38 d_{n z} R e_{l}^{0.25} W e_{l}^{-0.32}\left(\frac{\mu_{l}}{\mu_{g}}\right)^{0.37}\left(\frac{\rho_{l}}{\rho_{g}}\right)^{-0.47} \\
S M D=8.7 d_{n z}\left(R e_{l} W e_{l}\right)^{-0.28}
\end{gathered}
$$

Equations (6) and (7) are related to the injection pressure rather than inlet velocity derived by injected droplet measurement into stagnant air. The equation by Hiroyasu and Kadota considers the volumetric flow rate of the liquid, but does not consider any liquid properties. Thus the equation is not strictly valid for liquids with physical characteristics like the lubricant. Equation (7), instead, considers liquid density, surface tension, kinematic viscosity, air density and injection pressure, which is the most suitable approach for the modelled lubricant spray. Equation (8) is suitable for incomplete spray, in fact, the atomization process depends mainly on the injection velocity in the nozzle hole. The spray cannot be formed correctly (incomplete spray) for low injection velocities, causing an insufficient atomisation, with a long transformation process from liquid column to droplets. This is the case that can occur in the lube oil spray, which can be characterized from low velocity and laminar flow due to the high viscosity of the liquid phase. However, to describe the complete spray, equation (9) appears to be the most suitable one. [4]

At this stage, a computation of the injected mass, $m_{i n j}$, for each time-step is performed with equation

$$
m_{i n j}=k_{v} A_{n z} \sqrt{2 \Delta p \cdot \rho_{l}} \mathrm{dt}
$$

Using the computed SMD provides the possibility to estimate the Reynolds field of the mean droplet as a function of time. Thus, according to the studies of Desantes et al [8] [9], who presented a description of the drag coefficient $C_{d}$ for sprays, equation (11) has been used to calculate the drag force:

$$
\begin{cases}C_{d}=\frac{24}{R e}\left(1+0.15 R e^{0.687}\right) & \text { if } R e \leq 10^{3} \\ C_{d}=0.44 & \text { if } \operatorname{Re}>10^{3}\end{cases}
$$

Finally, It is possible to calculate the mean force acting on the jet, as a weighted arithmetic mean to the injected masses of the j-th drag forces.

So far, the dynamic characteristics of the spray has been considered. Now, it is necessary to make an estimation of the spray angle which will be applied to the definition of the $3 \mathrm{~d}$ behaviour. The approach followed was to use a literature expression of the spray cone angle and to correct it introducing the dependencies from the viscosity to make a best fit to the value measured during the tests. Considering the equation for the spray angle provided by [10], corrected with a nozzle length-diameter ratio provided by [11], a best fit relation (12) for the cone spray angle has been found:

$$
\vartheta=457 \cdot d_{n z}^{0.512} v_{l}^{-1.53} \operatorname{atan}\left[\frac{1}{C_{n z}} 4 \pi \frac{\sqrt{3}}{6}\left(\frac{\rho_{g}}{\rho_{l}}\right)^{0.5}\right]
$$

With

$$
C_{n z}=3+0.28 \frac{L_{n z}}{d_{n z}}
$$

The source code of this numerical simulation tool is based on Labview for a full integration with the previously exhibited neural network code and with the designed graphical user interface (GUI). Main details and most important features of the simulation tool are shown in Fig. 8 


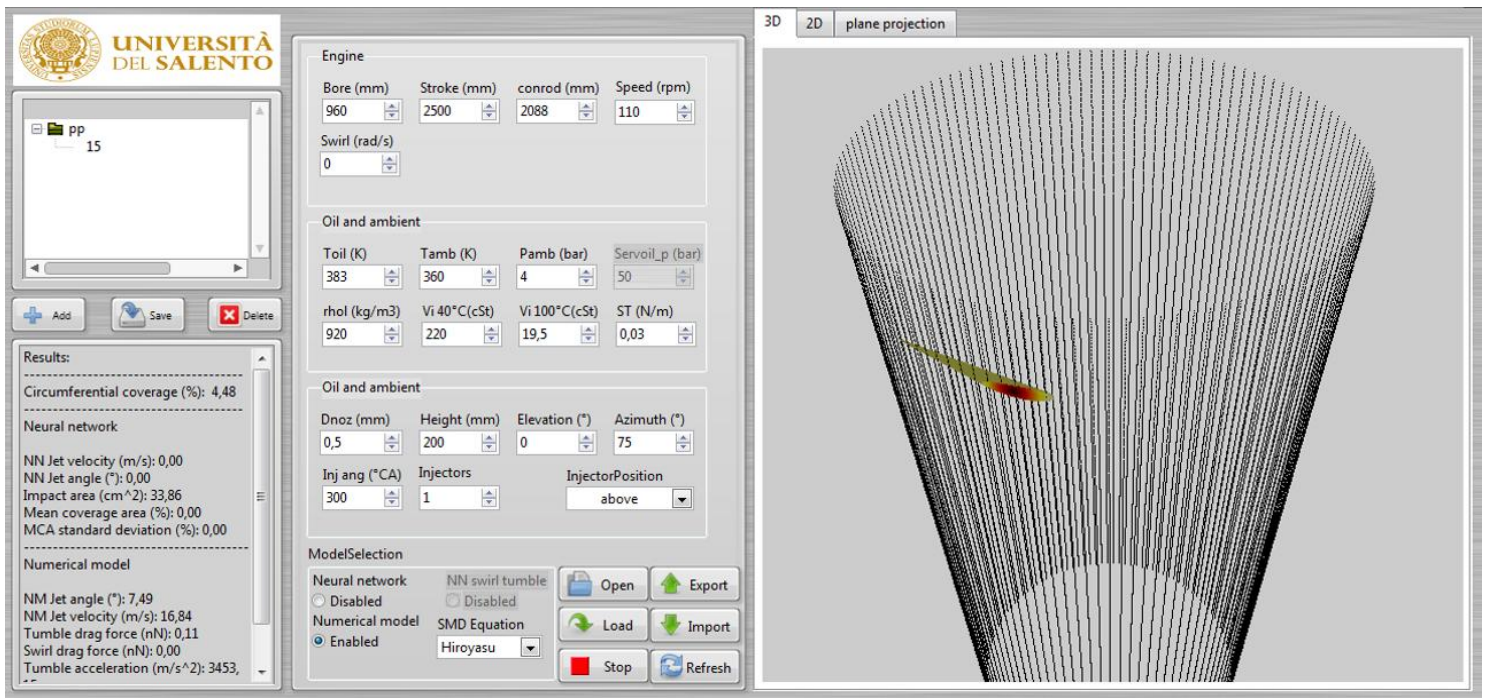

Fig.8: Main screen of the Lube oil simulation tool [4]

The main screen of the application is split into three main parts:

- Tree of configuration and calculation results

- Input parameters and computation model selection. In this mask, it is possible to insert the geometrical engine specifications (bore diameter, stroke, etc...), lubricant properties and injection system properties

- The graphical section shows a series of three tabs for the visualization in 3d render and on a 2D plane of the performed computation.

The developed application is capable of predicting the performance of a complete lubrication system, composed by a number of up to eight lubricant injectors with a maximum of five holes per injector. [4]

Another feature of the new simulation tool relates to the prediction of the shape of the lube oil spray. Fig. 9 compares the predicted spray characteristics with the measured ones of the above investigated ambient conditions at $25 \%$ and $100 \%$ engine load equivalent system conditions.

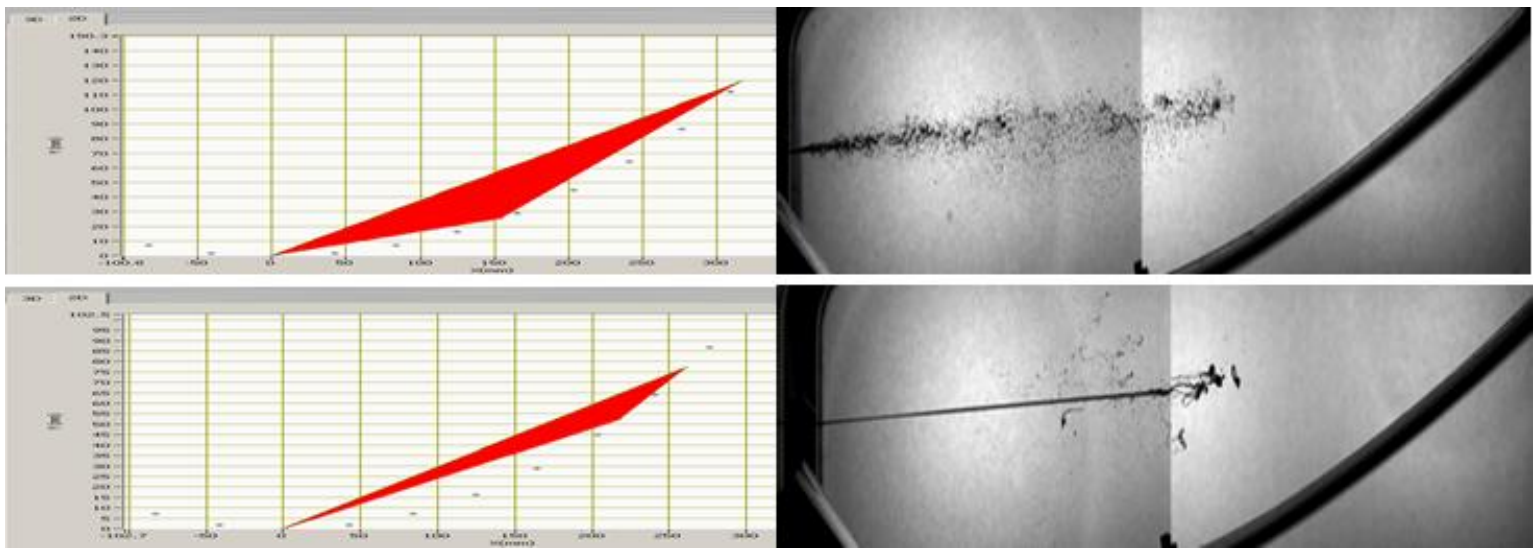

Fig.9: Comparison between predicted and measured lube oil injection spray characteristics for $25 \%$ engine load equivalent system conditions (1,6 bar of absolute cell pressure and $421 \mathrm{~K}$ lube oil temperature) and 100\% engine load equivalent system conditions (4.8 bar of absolute cell pressure and $476 \mathrm{~K}$ lube oil temperature)

Next steps of this investigation yield the implementation of experimentally gained data in a neuronal network of the simulation tool in order to enhance predictive accuracy of the model and to support design optimization of the new lubrication system development. 


\section{Conclusions}

The analysis presented here highlights key steps towards the development of a new, common rail based lubrication system comprising the application of needle lift type lubricant injectors as well as the consequent application of a dedicated testing facility and furthermore focuses on relevant simulation tool development activities in order to provide all the required tools for a successful lubrication system development. The simulation of relevant injector kinetics allows to predict lubricant flow characteristics in the injector and highlights the optimization potential of such an approach. A distinct testing facility supports design variation investigations of relevant lubrication system components under close to real engine boundary conditions and the same time provides the possibility to validate a new lubricant spray and impingement simulation tool. The combination of all activities represents a sound basis for further developments and optimization cycles in order to obtain the desired lubrication performance.

\section{Acknowledgements}

This project has received funding from the European Union's Horizon 2020 research and innovation programme under grant agreement No 634135

$\begin{array}{ll}\text { Nomenclature } & \\ \Delta p: & \text { pressure drop across the nozzle hole } \\ \rho_{g} \text { and } \rho_{l}: & \text { gas and liquid density respectively } \\ V_{f}: & \text { injected volume of liquid } \\ \sigma: & \text { liquid/air surface tension } \\ v_{l}: & \text { kinematic viscosity of the liquid } \\ d_{n z}: & \text { nozzle diameter } \\ L_{n z}: & \text { nozzle length } \\ R e_{l}: & \text { Reynolds number of the liquid } \\ W e_{l}: & \text { Weber number of the liquid } \\ \mu_{l}: & \text { dynamic viscosity of the liquid } \\ \mu_{g}: & \text { dynamic viscosity of the gas phase } \\ d_{n z}: & \text { nozzle diameter [mm]; } \\ v_{l}: & \text { lubricant viscosity [cSt]; } \\ \rho_{g} \text { and } \rho_{l}: & \text { the gas and liquid density respectively } \\ C_{n z}: & \text { nozzle geometry coefficient }\end{array}$

\section{References}

[1] P. Harper, M. Stark, Measurement of Piston Ring lubricant Film Thickness in Fired Engine using Ultrasonic Reflectometry, Cimac Paper, 2013

[2] M. Stark, R. Mittler, Lubricant Flow Optimization of Large Two Stroke Marine Diesel Engine Piston Ring Packs, ICEF2016-9326, Proceedings of the ASME 2016 INternal Combustion Fall Technical Conference

[3] M. Stark, R. Mittler, Optimization of Tribodynamic Effects to improve the Reduction Potential of Particulate Matter Concentrations in the Exhaust Gas of Large Two Stroke Marine Diesel Engines, 14FFI-0396, SAE2014

[4] M. Giangreco, Numerical and experimental study of two stroke marine engine lubrication systems, pHD thesis, University of Salento, 2013"

[5] A. de Risi, "Evaluation of Piston Ring Pack Phenomena," University of Salento - Internal report

[6] N. Ashgriz, "Handbook of atomization and sprays-Theory and applications," Springer, 2011, p. 101

[7] J. B. Heywood, "Internal combustion engine fundamentals," McGraw-Hill, 1988

[8] R. D. Reitz and F. V. Bracco, "On the Dependence of Spray Angle and Other Spray Parameters on Nozzle Design and Operating Conditions," SAE paper, no. 790494, 1979

[9] H. Hiroyasu and T. Katoda, "Fuel Droplet Size Distribution in a Diesel Combustion Chamber," SAE Trans, no. Paper 74017, 1974

[10] M. M. Elkotb, "Fuel Atomization for Spray Modeling," Prog. Energy Combust. Sci., vol. 8, no. 1, pp. 61-91, 1982

[11] H. Hiroyasu, M. Arai and M. Tabata, "Empirical Equations for the Sauter Mean Diameter of a Diesel Spray," SAE Technical Paper, no. 890464, 1989

[12] J. M. Desantes, X. Margot, J. M. Pastor, M. Chavez and A. Pinzello, " CFD-Phenomenological diesel spray analysis under evaporative conditions," Energy Fuels, vol. 23, p. 3919-3929, 2009

[13] Lockhart, R.W., Martinelli, R.C.; Chem. Eng. Prog., Vol. 45. 1949, pp. 39-48

[14] Josef Kunes: Dimensionless Physical Quantities in Science and Engineering. Elsevier, 2012, ISBN 0-12391458-2, S. 263

[15] I. Parson (ed.), Fieldspars and Their Reactions, 449 - 499, 1994 Kluwer Academic Publishers 